\title{
Receptor-Interacting Protein 140 Orchestrates the Dynamics of Macrophage M1/M2 Polarization
}

\author{
Yi-Wei Lin Bomi Lee Pu-Ste Liu Li-Na Wei \\ Department of Pharmacology, University of Minnesota Medical School, Minneapolis, Minn., USA
}

\author{
Key Words \\ Receptor-interacting protein $140 \cdot$ Macrophage \\ polarization - Endotoxin tolerance - Wound healing · \\ Inflammation · PTP1B · STAT6
}

M1 enhancer and the second, in the cytosol, is an M2 suppressor. Modulating the level and/or subcellular distribution of RIP140 can be a new therapeutic strategy for diseases where inflammatory/anti-inflammatory responses are critical.

๑) 2015 S. Karger AG, Basel

\section{Introduction}

Macrophages are innate immune cells and play a central role in inflammation and host defense; they are highly heterogeneous and exhibit diverse functional phenotypes in response to different environmental cues $[1,2]$. In general, they undergo two types of polarized activation, classic proinflammatory activation (M1) and alternative anti-inflammatory activation (M2) [3, 4]. The M1 macrophage produces high levels of proinflammatory cytokines and reactive nitrogen and oxygen intermediates, and it generally harbors strong microbicidal and tumoricidal activities. The M2 macrophage is anti-inflammatory and primarily involved in resolving inflammation, parasite containment and tissue remodeling. When tissues are damaged following infection or injury, inflammatory monocytes are recruited from the circulation and differ-

Y.-W.L. and B.L. contributed equally to this work.

\section{KARGER}

E-Mail karger@karger.com

www.karger.com/jin
(C) 2015 S. Karger AG, Basel

1662-811X/15/0081-0097\$39.50/0
Dr. Li-Na Wei

Department of Pharmacology, University of Minnesota Medical School 6-120 Jackson Hall, 321 Church St. SE

Minneapolis, MN 55455 (USA)

E-Mail weixx009@umn.edu 
entiated into macrophages as they migrate into the affected tissues. These recruited macrophages often first show an M1 phenotype, producing proinflammatory cytokines such as interleukin-1 (IL)-1 and tumor necrosis factor a (TNFa). Subsequently, the recruited macrophages then shift to become more M2-like, producing regulatory cytokines such as IL-10 and transforming growth factor $\beta$ (TGF $\beta$ ). It remains unclear whether the M1-M2 phenotype switch occurs in the same macrophage, but it is widely accepted that M1-M2 switch among macrophage populations is an essential process during recovery [5]. This macrophage phenotype switch must be tightly regulated to maintain the homeostasis of innate immunity. Uncontrolled macrophage polarization is frequently implicated in diseases such as sepsis, autoimmune diseases and cancers $[3,6-8]$. Cytokines that stimulate the M1 or M2 phenotype have been largely uncovered and principal transcription factors for M1 versus M2 activation have also been well-characterized [9-12]; however, whether the dynamics of the M1-M2 switch or the homeostatic control of this phenotypic switch involve cell-autonomous factors in macrophages is unknown.

Upon infection, inflammation occurs as a defense mechanism, but uncontrolled inflammation leads to dangerous pathological states including septic shock [13, 14]. An important host adaptation to protect against septic shock is to establish a state of 'endotoxin tolerance' (ET), where macrophages become transiently unresponsive and fail to produce inflammatory cytokines upon further endotoxin challenge $[15,16]$. Molecules that may mediate ET by suppressing Toll-like receptor (TLR) signaling have been reported [17-19]. Recently, we have reported that a nuclear coregulator, receptor-interacting protein 140 (RIP140) [20], which is involved in various physiological processes [21], also plays a crucial role in the establishment of ET [22]. We found that RIP140 is the principal nuclear coactivator of NF- $\mathrm{KB}$ in endotoxin-challenged M1 macrophages and that upon endotoxin challenge, it is rapidly degraded by Syk-initiated ubiquitination, which renders NF- $\mathrm{KB}$ inactive [22]. Thus, reducing the RIP140 protein level facilitates ET and protects against septic shock [22, 23]. Other studies have shown that endotoxin-challenged M1 macrophages, while unable to respond to further endotoxin stimulation, can still respond to stimuli for M2 polarization [24, 25]. Is a state of ET required for proceeding to M2 polarization? Are there cellular factors regulating the progression of stimulated macrophages from M1, ET and then M2? These are central questions to be addressed in this study.

Numerous disease conditions involve the coordination of inflammatory and anti-inflammatory responses
[26]. Wound healing encompasses macrophage M1 polarization and transition to a state of ET and then to an M2 phenotype. When this transition is out of control, macrophages could be locked into a specific phenotype, leading to chronic inflammation and tissue destruction. In this study, we uncover that RIP140 is not only crucial for M1-ET establishment, but can also negatively regulate the progression of a macrophage switch to an M2 state during recovery. Interestingly, this particular function of RIP140 in M2-stimulated macrophages is executed by its cytosolic form, which activates a specific cytosolic phosphatase that inactivates the master regulator of M2 polarization, pSTAT6. We employ both in vitro and in vivo wound-healing models to demonstrate the dual functions of RIP140 in regulating the dynamics of the macrophage M1-M2 phenotypic switch and to explain, at least partially, the plastic nature of macrophage polarization in various disease conditions.

\section{Materials and Methods}

Information about reagents and general procedures for cell culture, virus production, immunofluorescence, gene expression, ChIP, animals, antibodies and in vitro wound healing are provided as online supplementary material (for all online suppl. material, see www.karger.com/doi/10.1159/000433539).

\section{Flow Cytometry}

Macrophage surface antigens were blocked with Fc block (BD Biosciences), incubated with fluorophore-conjugated or isotype control antibodies for $1 \mathrm{~h}$, washed and centrifuged at $500 \mathrm{~g}$ for $5 \mathrm{~min}$ and resuspended for FACSCalibur analyses using WinMDI software. The gated $\mathrm{CD} 11 \mathrm{~b}^{+} \mathrm{F} 4 / 80^{+}$cells were examined with antiCD11c (as M1) or anti-CD206 (as M2) antibody.

\section{In vitro Phosphatase Activity Assay}

RAW264.7 cell lysate was prepared in $50 \mathrm{mM}$ HEPES, $0.1 \mathrm{~mm}$ EGTA, $0.1 \mathrm{~mm}$ EDTA, $120 \mathrm{mM} \mathrm{NaCl}$ and $0.5 \% \mathrm{NP}-40$, incubated with immobilized PTP1B capture antibody-bound microplates and washed, and then synthetic phosphopeptide substrate was added. The generated free phosphate was detected using malachite green and molybdic acid with absorbance at $620 \mathrm{~nm}$.

\section{In vivo Wound-Healing Assay}

Cutaneous wounds were made on both sides of the shaved back ( 2 wounds per mouse), with a $5-\mathrm{mm}$, round biopsy punch under anesthesia. The wound size was recorded daily and analyzed by Image J.

\section{Statistical Analysis}

Experiments were performed at least twice and presented as means \pm SD. One-way ANOVA or Student's t test was used. $p$ values $\leq 0.05$ were considered statistically significant $(* \mathrm{p}<0.05$; ** $\mathrm{p}<0.01$; *** $\mathrm{p}<0.001)$. 


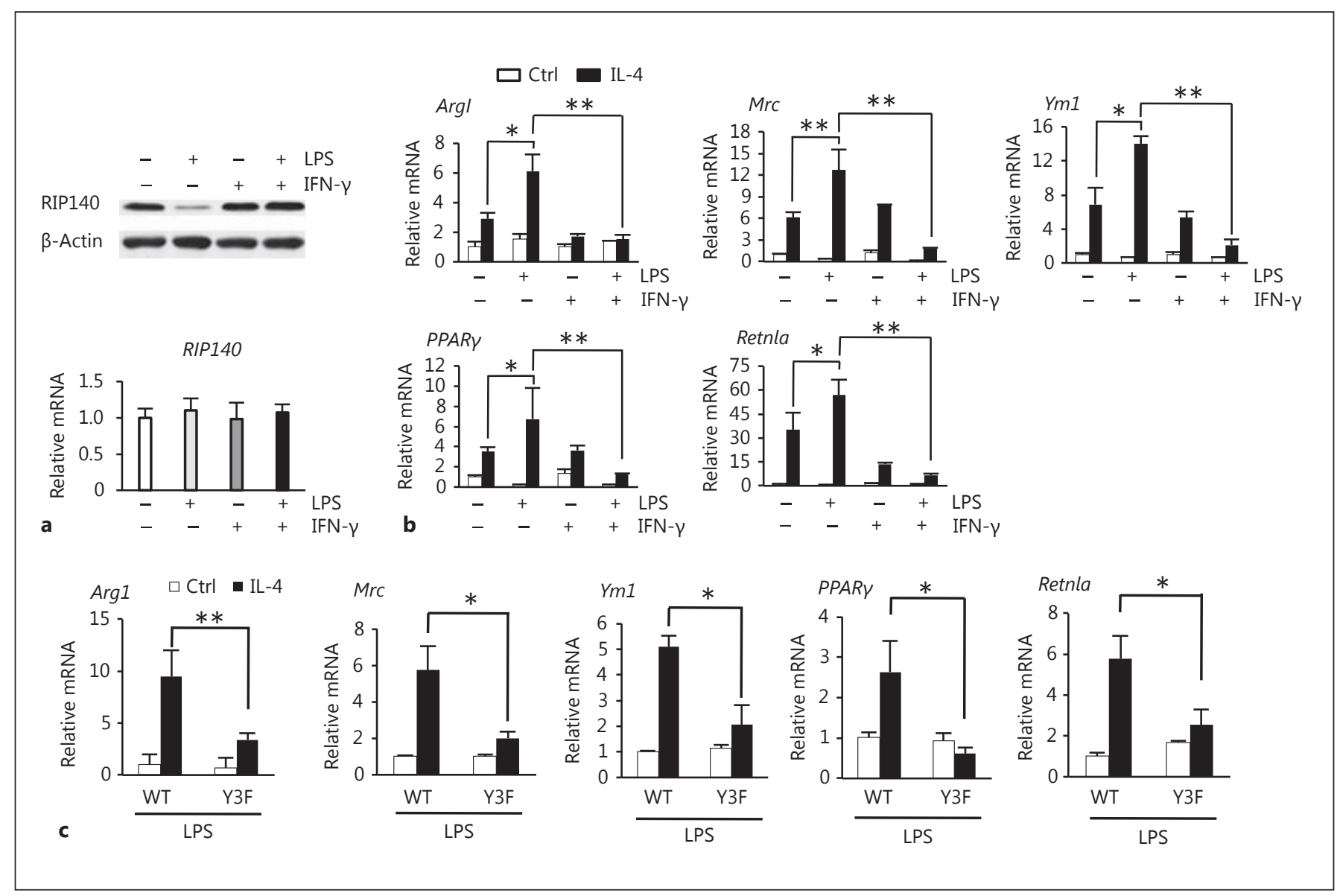

Fig. 1. ET established by degrading RIP140 facilitates macrophage M2 polarization. a Western blot (top) and qPCR (bottom) of RIP140 in primary PM with or without IFN- $\gamma$ pretreatment for $16 \mathrm{~h}$ followed by LPS ( $10 \mathrm{ng} / \mathrm{ml}$ ) treatment for $24 \mathrm{~h}$. b qPCR of M2 marker expression in PM with or without IFN- $\gamma$ pretreatment followed by LPS stimulation, and then treated with (black bars) or without (open bars) IL-4 for $6 \mathrm{~h}$. Results are presented relative to control (neither LPS nor IFN- $\gamma$ ). c qPCR of M2 marker expression in lentinvirus-WT-RIP140 (WT) or lentivirus-Y3F-RIP140 (Y3F) infected (for $48 \mathrm{~h}$ ) PM that were then challenged with LPS for $18 \mathrm{~h}$, followed by control or IL-4 stimulation for $6 \mathrm{~h}$. Results are presented relative to the control. Data are representative of 3 experiments (means $\pm \mathrm{SD})$, and Student's t test $(\mathrm{n}=3)$ was used. ${ }^{*} \mathrm{p}<0.05 ;{ }^{* *} \mathrm{p}<0.01 ;{ }^{* * *} \mathrm{p}<0.001$. Ctrl = Control.

\section{Results}

Failure to Establish RIP140 Degradation-Mediated ET Prevents Macrophage M2 Polarization

We previously showed that RIP140 is required for macrophage M1 polarization, but it must then be rapidly degraded in order to establish ET and prevent inflammatory storm such as sepsis. In this study, we first found that under the condition when ET fails to be established and RIP140 cannot be degraded, e.g. following stimulation with IFN- $\gamma$ [27], subsequent M2 polarization is severely dampened. Figure 1a shows that, as predicted, in an LPStreated M1 macrophage, RIP140 protein is degraded without altering its mRNA level, which is abolished in
IFN- $\gamma$ pretreated cells. Figure $1 \mathrm{~b}$ shows that IL-4-stimulated M2 polarization, as shown in LPS-tolerated cells, is dramatically dampened in IFN $-\gamma$ pretreated cells. We speculated that RIP140 might play a regulatory (negative) role in macrophage polarization for the transition to M2 and, to test this possibility, we employed a nondegradable mutant, RIP140 (Y3F; Tyr364, Tyr418 and Tyr436 all mutated into phenylalanine to prevent degradation; online suppl. fig. S1) [22]. Figure 1c shows that expressing this nondegradable RIP140 (Y3F) but not the wild-type RIP140 (WT), indeed significantly diminishes the effect of IL-4-stimulated M2 polarization; this is supported by the significantly reduced expression of M2 markers. These results demonstrate a pivotal role for RIP140, not 

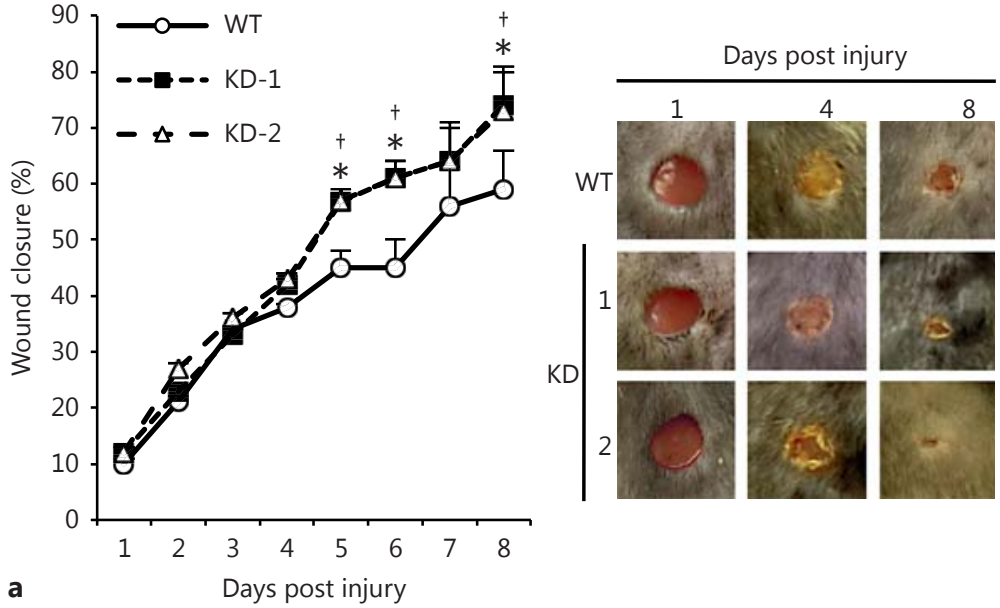

a
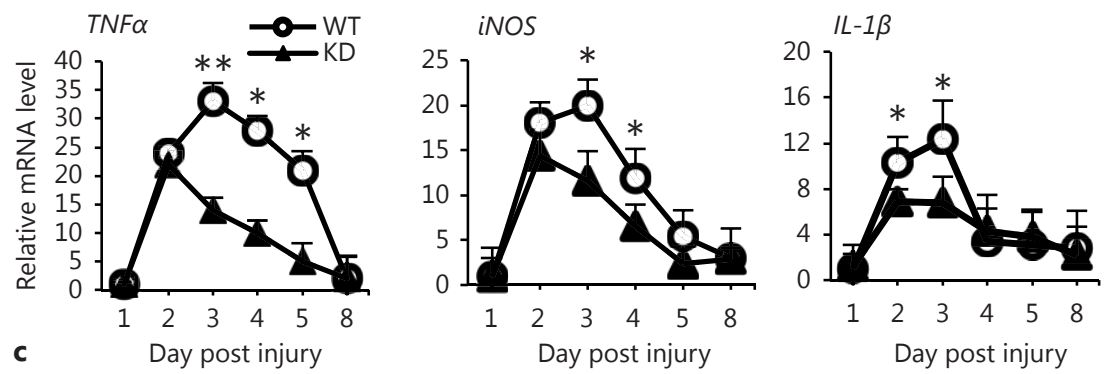

b
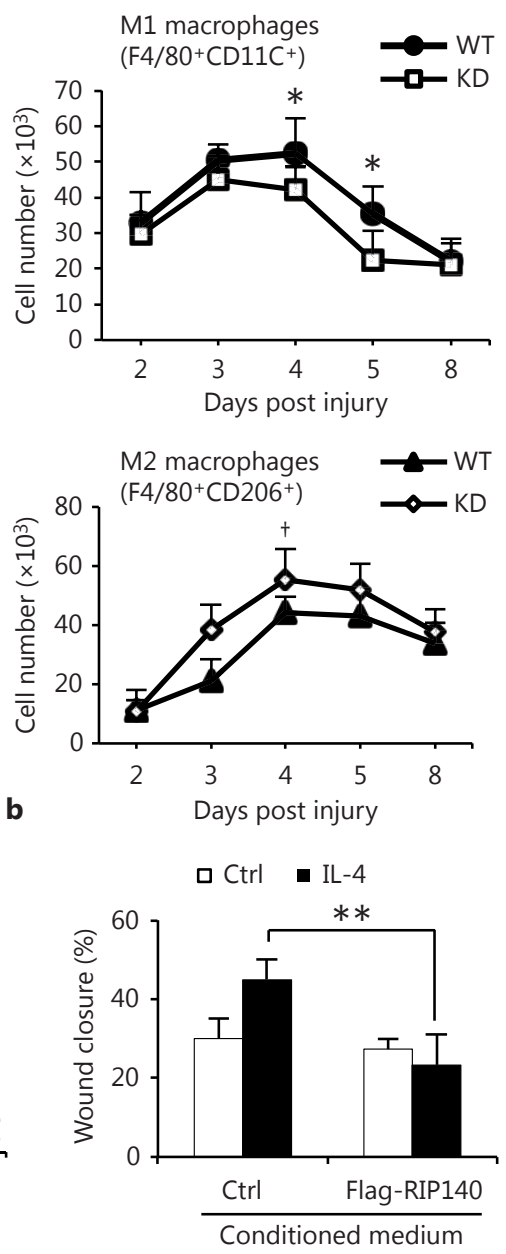

口 Ctrl $\quad \mathrm{IL}-4$
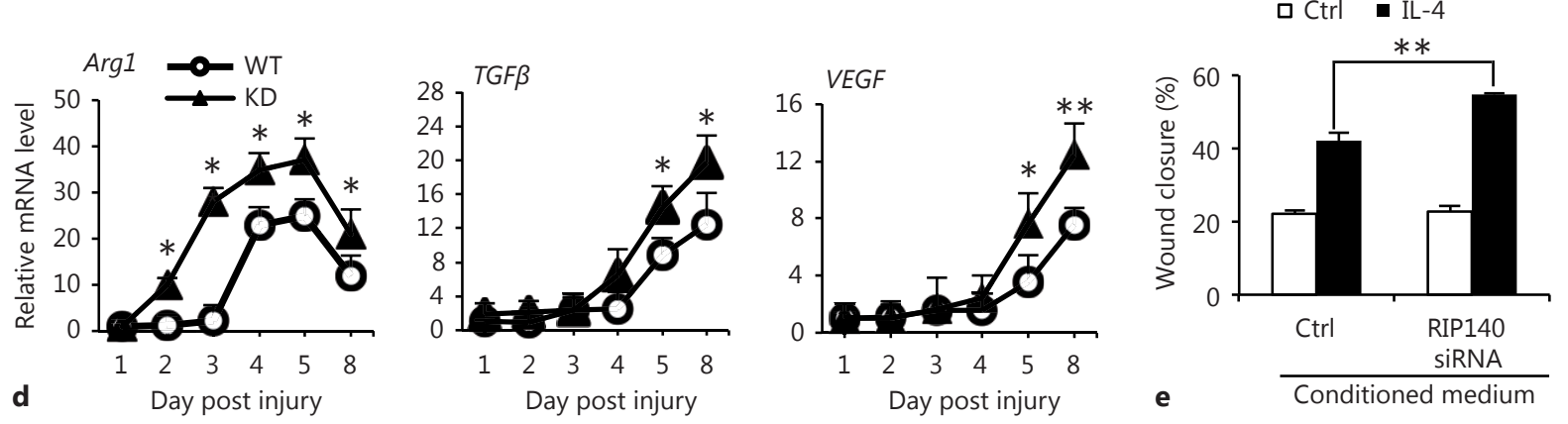

Fig. 2. Reducing RIP140 level promotes wound healing by facilitating M1-M2 switch. a Daily record of wound closure on WT and MФRIP140KD mice of 3 experiments of 2 independent MФRIP140KD lines (means \pm SD). A one-way ANOVA test was used ${ }^{*} \mathrm{p}<0.05$ (WT vs. KD-1); ${ }^{\dagger} \mathrm{p}<0.05$ (WT vs. KD-2). b FACS evaluation of $\mathrm{M} 1\left(\mathrm{~F} 4 / 80^{+} \mathrm{CD} 11 \mathrm{C}^{+}\right.$, top) and M2 (F4/80 ${ }^{+} \mathrm{CD} 206^{+}$, bottom) populations in the wounds of 3 experiments. M1 and M2 numbers are normalized to wound mass (cell numbers $/ \mathrm{mg}$ ). Student's $\mathrm{t}$ test $(\mathrm{n}=3)$ was used. ${ }^{*} \mathrm{p}<0.05$ (WT-M1 vs. KD-M1); ${ }^{\dagger} \mathrm{p}<0.05$ (WT-M2 vs. KD-M2). c, d qPCR of M1 and M2 marker expression in the wounds. e In vitro wound healing results of 3 experiments. Student's t test $(\mathrm{n}=3)$ was used. ${ }^{*} \mathrm{p}<0.05$; ${ }^{* *} \mathrm{p}<$ 0.01; ${ }^{* * *} \mathrm{p}<0.001$. Ctrl $=$ Control. 
only in promoting M1 polarization during an inflammatory response but also in controlling the phenotypic switch from the M1 to the M2 state.

\section{Reducing RIP140 Level Promotes Wound Healing by} Facilitating M1-M2 Switch

The macrophage M1-M2 phenotype switch is critical for wound healing. We asked whether and how changing RIP140 levels might affect wound-healing efficiency. We first employed a macrophage-specific RIP140 knockdown (MФRIP140KD) mouse as an in vivo model [22] in which the RIP140 protein level in monocyte-macrophage populations is reduced for $90-95 \%$ (online suppl. fig. S2). As shown in fig. 2a, wound closure in MФRIP140KD mice (2 independent lines: KD-1 and KD-2) is more efficient than that in WT mice. We then examined the dynamics of M1-M2 switch during the wound-healing process (fig. 2b). In WT mice (closed markers), M1-M2 switch occurs on approximately the 5th day after the wound whereas in MФRIP140KD KD (open markers), M1-M2 switch occurs on approximately the 3rd day. Consistently, in the wounds, the levels of all the examined M1 marker genes (TNF $\alpha$, iNOS and $I L-1 \beta$ ) are lower in MФRIP140KD mice than in WT mice (fig. 2c) whereas the levels of all the examined M2 marker genes (Arg1, $T G F \beta$ and VEGF) are significantly elevated in MФRIP140KD mice (fig. 2d). These data support the notion that the RIP140 protein level is an important factor that regulates the kinetics of macrophage phenotypic switch in, and thus the efficiency of, the wound-healing process.

We then utilized an in vitro primary macrophage (PM) culture to examine the effects of altering RIP140 protein levels in M2 polarization (online suppl. fig. S3a). Using lentivirus to overexpress or knock down RIP140 in primary PM, we found that IL-4 induction of all characteristic M2 genes is significantly attenuated by overexpressing RIP140, but is significantly elevated by knocking down RIP140. Further, the activity of arginase 1 (Arg1), a critical factor in M2 macrophages, is significantly elevated by knocking down the RIP140 level, and is reduced by elevating the RIP140 level (online suppl. fig. S3b). We further employed an in vitro wound-healing model of 293TN monolayer cultures supplemented with conditioned media of macrophage cultures, where the RIP140 level had been altered (fig. 2e; online suppl. fig. S4). It appears that the conditioned medium of the RIP140-overexpressing, IL-4-treated macrophage culture suppresses healing in the wounded 293TN culture (fig. 2e: top panel; online suppl. fig. S4: left panel) whereas the conditioned

RIP140 Orchestrates Macrophage M1/M2 Polarization medium of RIP140 knockdown, IL-4-treated macrophage culture significantly improves wound closure (fig. 2e: bottom panel; online suppl. fig S4: right panel). These results support that RIP140 regulates the macrophage M1-M2 switch to affect wound healing both in vivo and in vitro.

\section{Preventing RIP140 Degradation Delays Wound \\ Healing}

We next examined whether preventing RIP140 degradation in macrophages would delay wound healing in vivo. In order to specifically monitor the effects elicited by disturbing the dynamics of macrophage polarization in adult stages, we employed bone marrow transplantation to replace the animal's endogenous macrophages with myeloid cells carrying either green fluorescent protein (GFP) only, WT RIP140-GFP or the nondegradable mutant Y3F RIP140-GFP, and examined their woundhealing processes. Comparable BTM efficiency was validated (online suppl. fig. S5). It appears that wound healing in mice receiving myeloid cells carrying WT RIP140 is slightly delayed compared to those receiving GFP only (control), but complete healing (with 100\% wound closure) occurs on day 12 for both groups. Interestingly, wound closure in mice receiving Y3F-RIP140 is significantly less efficient; by day 12 , their wounds close for only $70 \%$ (fig. 3a). Further, in the wounds of the Y3F-RIP140 group, the M1 population is expanded but the M2 population is reduced, when compared to the WT RIP140 group (fig. 3b). Consistently, in the wounds of the Y3FRIP140 group, in the course of healing, the expression of all the monitored M1 marker genes (TNF $\alpha$, $i N O S$ and $I L$ $1 \beta)$ is significantly elevated and the expression of all the monitored M2 marker genes ( $\operatorname{Arg} 1, T G F \beta$ and $V E G F)$ is significantly lowered (fig. 3c, d). These data further validate the notion that elevating the RIP140 protein level, e.g. by blunting its degradation in monocytes/macrophages during injury, facilitates and locks these macrophages in their M1 state, resulting in an impaired woundhealing process.

RIP140 Suppresses M2 Macrophage Polarization by Inhibiting the Activation of STAT6 (Phosphorylation) in the Cytosol

We noticed that the suppressive effect of RIP140 on M2 gene expression is evident only after treatment with M2 cues such as IL-4 (online suppl. fig. S3), indicating that RIP140 targets the downstream signaling pathway of IL-4. Interestingly, in IL-4-stimulated cells, endogenous RIP140 is gradually translocated from the nucleus to the 
Fig. 3. Preventing RIP140 degradation delays wound healing by inhibiting M1-M2 switch. a Daily record of wound closure in mice receiving WT-GFP-, WT-RIP140- or Y3F-RIP140-expressing cells. A one-way ANOVA test was used * $\mathrm{p}<0.05$; ${ }^{* *} \mathrm{p}<$ 0.01 . b FACS analyses of M1 and M2 populations normalized to the wound mass (cell numbers/mg). c, $\mathbf{d}$ qPCR of M1 and M2 marker expression in the wounds of 3 experiments. Student's t test $(n=3)$ was used. ${ }^{*} \mathrm{p}<0.05$; ** $\mathrm{p}<0.01$; *** $\mathrm{p}<0.001$ (WTRIP140 vs. Y3F-RIP140). Ctrl = Control.
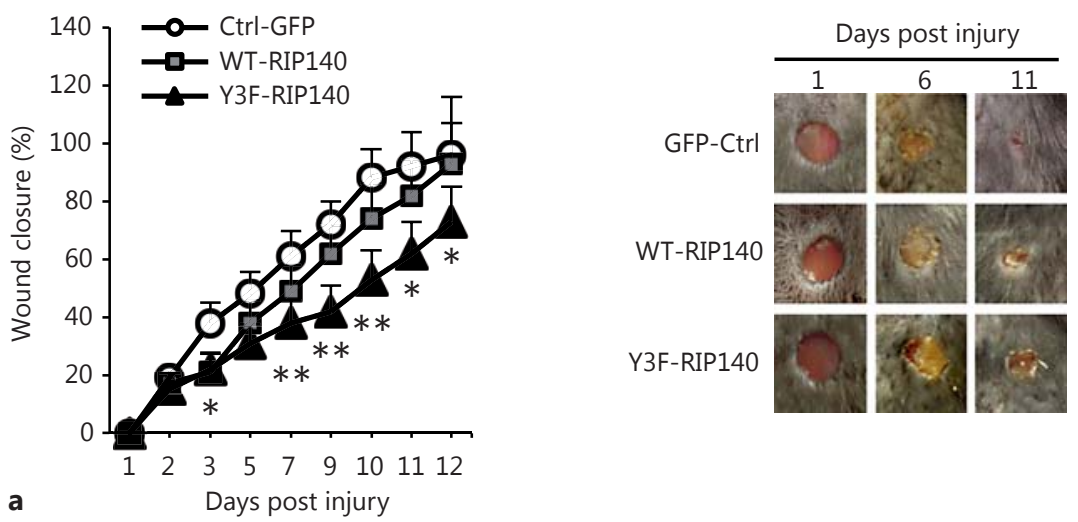

a

口 Ctrl-GFP
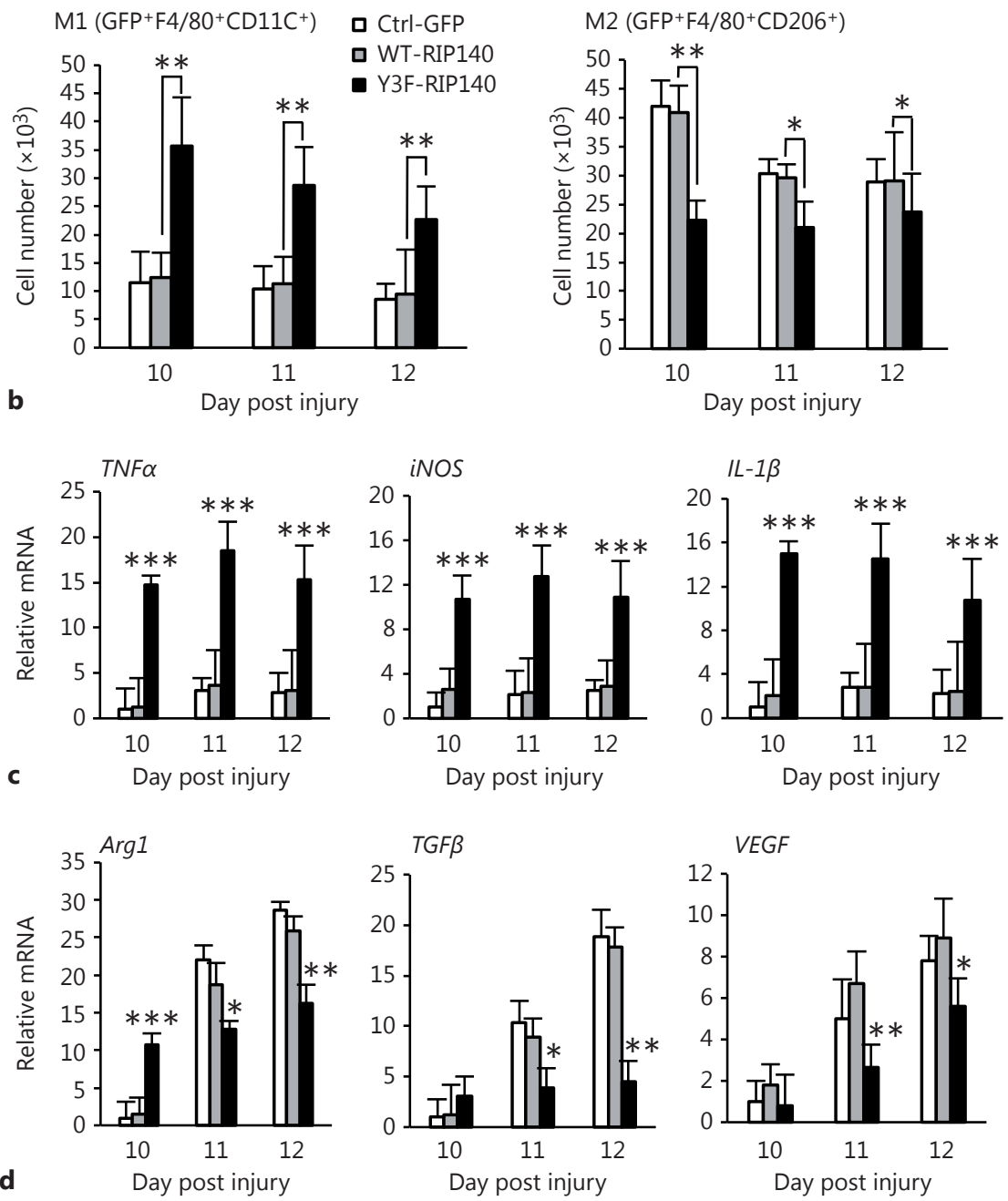


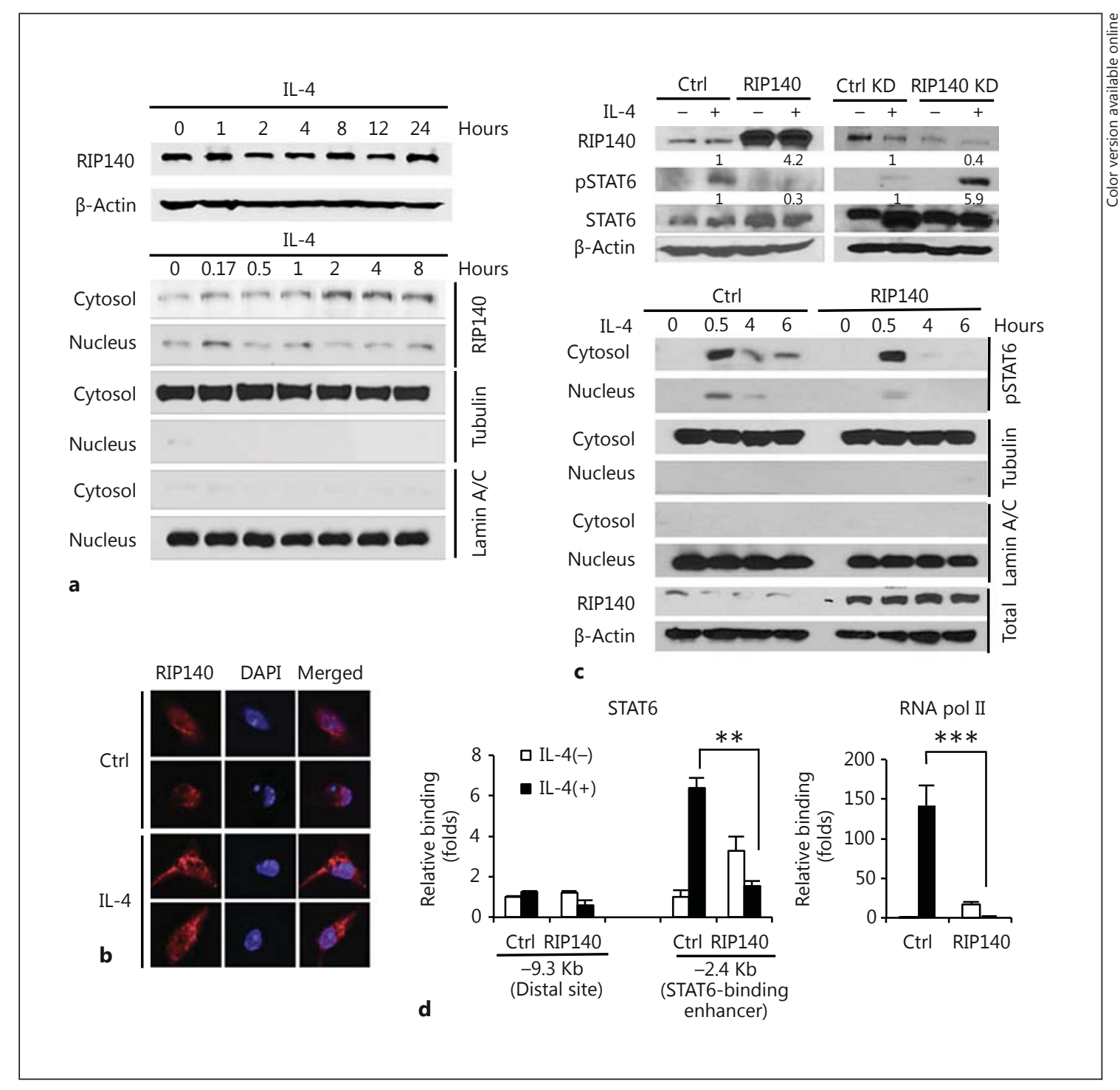

Fig. 4. RIP140 translocates to the cytosol and suppresses M2 marker gene expression via reducing STAT6 phosphorylation. a Western blot of endogenous RIP140 from total (top) and the nuclear and cytosolic (bottom) fractions in IL-4-treated RAW264.7. b Immunofluorescence of endogenous RIP140 (red) in IL-4-treated (10 $\mathrm{ng} / \mathrm{ml})$ RAW264.7. c Western blot of pSTAT6 and STAT6 in RIP140-overexpression (RIP140) or knockdown (RIP140 KD)

cytosol (fig. 4a, b); this appears to also be true for the RIP140-Y3F mutant protein (online suppl. fig. S6). The nuclear-cytosolic translocation of RIP140 also occurs in macrophages stimulated with other anti-inflammatory cytokines such as IL-13 (online suppl. fig. S7). To understand how RIP140 inhibits the IL-4 signaling pathway, we examined several potential targets and found that RIP140 suppresses the activation of the master signal transducer
RAW264.7, with or without IL-4 treatment (top). Western blot of pSTAT6 and STAT6 in the nuclear and cytosolic fractions (bottom) of RIP140-overexpressing RAW264.7 with IL-4 treatment. d ChIP of STAT6 and RNA pol II on Arg1 promoter in IL-4-treated RAW264.7. Data show 3 experimental results with Student's t test $(\mathrm{n}=3){ }^{*} \mathrm{p}<0.05$; $^{* *} \mathrm{p}<0.01{ }^{* * *} \mathrm{p}<0.001$. Ctrl $=$ Control.

for M2, STAT6 phosphorylation at Tyr641 (fig. 4c), resulting in its decreased DNA binding as well as RNA polymerase II recruitment to the promoter of its target genes like Arg1 (fig. 4d). These results indicate that upon IL-4 stimulation, RIP140 is translocated from the nucleus to the cytosol, where it suppresses the activation of the master regulator STAT6 (phosphorylation at Tyr641). M2 target gene expression is thus dampened. 


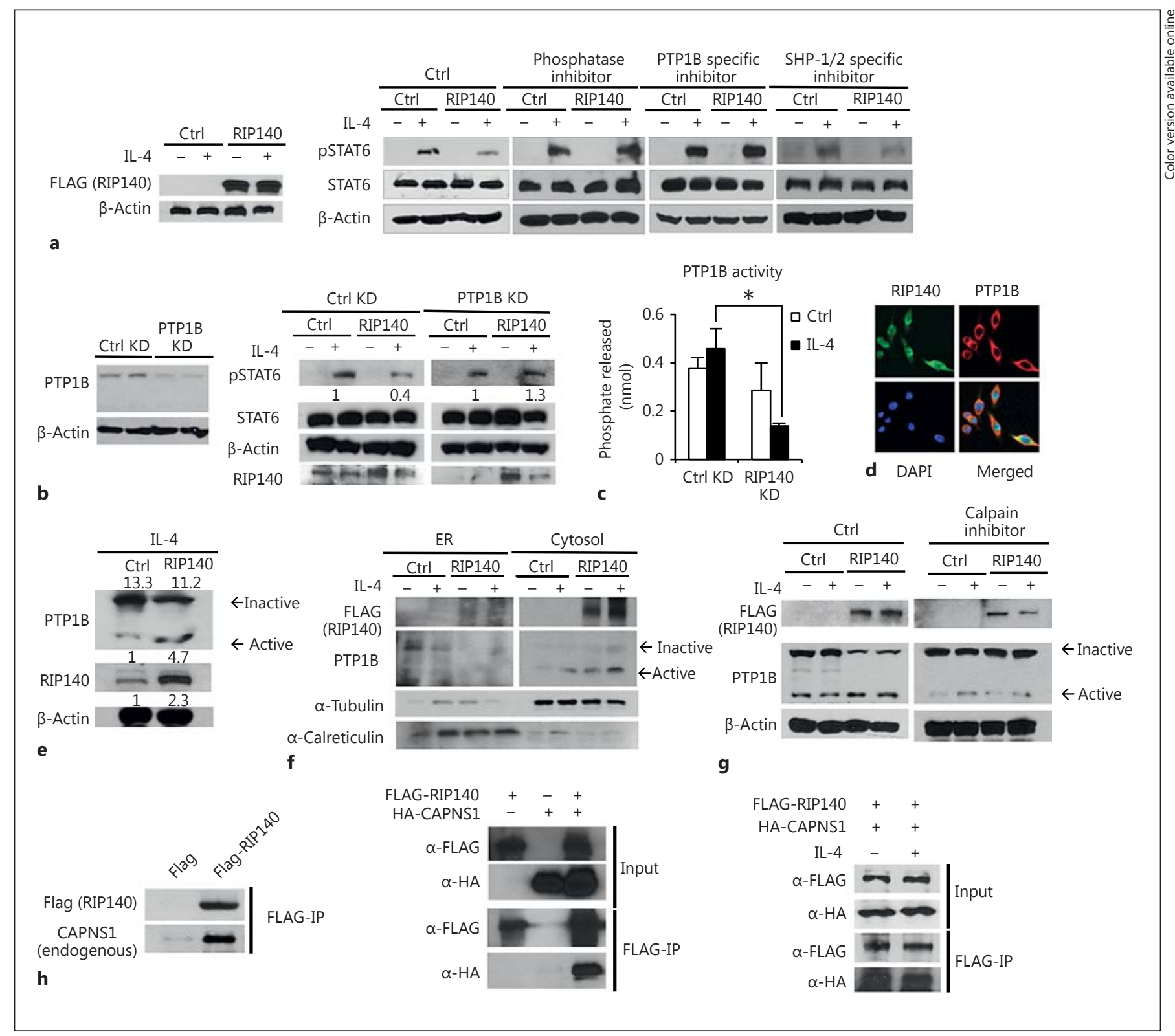

Fig. 5. RIP140 suppresses STAT6 phosphorylation by targeting calpain $1 / 2$ to activate PTP1B phosphatase. a Western blot of pSTAT6 and STAT6 in IL-4-treated control versus RIP140-overexpressing (left) RAW264.7 which were pretreated with a general phosphatase inhibitor, PTP1B specific inhibitor or SHP-1/2 specific inhibitor. b Western blot of PTP1B in control or PTP1Bknockdown RAW264.7 (left). Western blot of pSTAT6 and STAT6 in IL-4-treated control or RIP140-overexpressing RAW264.7 with control or PTP1B knockdown (right). c PTP1B phosphatase activity in RAW264.7 control or RIP140 knockdown, with or without IL-4 treatment. d Immunofluorescence of RIP140 (green), PTP1B (red) and DAPI (blue) in IL-4-treated RAW264.7. e Western blot of PTP1B in control or RIP140-overexpressing IL-4-treated PM. f Western blot of Flag-RIP140, PTP1B, tubulin and calreticulin in ER and cytosolic fractions of RAW264.7 control or IL-4-stimulated, with or without RIP140-overexpressing RAW264.7. g Western blot of PTP1B in the presence (right) or absence (left) of calpain inhibitor. $\mathbf{h}$ Co-ip of Flag-RIP140 and endogenous calpain regulatory subunit (CAPNS1) in IL-4-treated RAW264.7 (left), FlagRIP140 and HA-CAPNS1 in 293TN cells (middle), and FlagRIP140 and HA-CAPNS1 in IL-4-treated RAW264.7 (right), from 3 experiments. Student's t test $(n=3)$ was used. ${ }^{*} \mathrm{p}<0.05$; ${ }^{* *} \mathrm{p}<$ $0.01 ;^{* * *} \mathrm{p}<0.001$. Ctrl $=$ Control. 
RIP140 Targets Calpin1/2 to Activate PTP1B to

Reduce STAT6 Phosphorylation

To examine how RIP140 suppresses the activation of STAT6, i.e. its phosphorylation at Tyr641, we examined two possibilities - either impairing its kinase or enhancing its phosphatase activity, since RIP140 does not form a complex with STAT6 (data not shown). It appears that RIP140 does not affect the kinase, because silencing RIP140 still affects the induction of pSTAT6 even though kinases are blocked by inhibitors like staurosporine (online suppl. fig. S8a). Instead, it probably targets the dephosphorylation of pSTAT6. PTP1B has been reported as a phosphatase for pSTAT6 [28]. Indeed, both a general phosphatase inhibitor and a PTP1B-specific inhibitor, but not the SHP-1/2 specific inhibitor, block RIP140-triggered reduction in the pSTAT6 level (fig. 5a). The specificity of RIP140 to PTP1B is further confirmed, because silencing PTP1B abolishes the suppressive effect of RIP140 (fig. 5b). An in vitro phosphatase enzyme assay substantiated that RIP140 regulates PTP1B activity that inactivates (de-phosphorylates) pSTAT6 (fig. 5c) without affecting PTP1B mRNA levels (online suppl. fig. S8b).

We found that RIP140 and PTP1B are colocalized in the cytosol of IL-4-treated macrophages (fig. 5d). Further, the level of 'active' PTP1B, typically cleaved from its precursors on the ER membrane, is increased by overexpressing RIP140 (fig. 5e). We speculated that in IL-4-treated cells, RIP140 is exported to the cytosol and translocated to ER to facilitate the activation of PTP1B precursors, and performed ER and cytosol fractionation to examine the distribution of the active and inactive forms of PTP1B protein. Elevating the RIP140 level increases the active, cytosolic PTP1B and reduces the inactive, ER-associated PTP1B (fig. 5f), which supports that RIP140 enhances PTP1B activity by facilitating the cleavage of its precursor. The PTP1B precursor on the ER is cleaved by a calciumdependent protease, calpain. Interestingly, we found that RIP140 forms complexes with the calpain regulatory subunit, CAPNS1, in the presence or absence of IL-4 (fig. 5h). In addition, calpain inhibitor pretreatment completely blocks RIP140-regulated PTP1B cleavage in IL-4-treated M2 PM (fig. 5g). These data are consistent with previous reports that PTP1B is one of the targets of caplain $1 / 2$ [29, $30]$, and CAPNS1 is necessary for calpain $1 / 2$ activity [31, 32]. All together, the data demonstrate that in IL-4-treated macrophages, RIP140 is increasingly translocated from the nucleus to become more associated with the calpain regulatory subunit to regulate calpain $1 / 2$ activity, which cleaves PTP1B precursor from ER, releasing active PTP1B to inactivate pSTAT6 in the cytosol.

RIP140 Orchestrates Macrophage M1/M2 Polarization

\section{Discussion}

In this study, we used ET and wound-healing models to understand the regulation of M1/M2 polarization. While it has been reported that macrophages in the ET state exhibit M2-like features and are more sensitive to M2 cues, the underlying mechanism remained elusive [24, 25]. Here, we demonstrate that the level of RIP140 protein is crucial: (1) RIP140 suppresses the activation of the M2 master regulator STAT6 and (2) for inflammatory macrophages to proceed to a state of ET, the RIP140 level must be reduced. As such, ET macrophages generally have low RIP140 levels and are more sensitive to M2 activation.

As the wound healing progresses, the macrophage phenotype switches from M1 to M2 [5]. The transition from inflammatory M1 polarization to alternative antiinflammatory M2 polarization is critical for the healing process. One critical molecular event in this transition is the rapid reduction of the NF- $\kappa \mathrm{B}$ coactivator RIP140 protein level, which allows timely dampening of inflammation (via the inactivation of NF- $\kappa B$ ). Lowering the RIP140 level by rapid degradation is made possible by the action of a specific SCF E3 ubiquitin ligase that ubiquitinates RIP140 [22]. This study extends our previous finding and demonstrates a new function of RIP140, which is to regulate the dynamics of M1 versus M2 polarization. On the cellular level, M1-M2 transition requires the establishment of ET, at least for TLR-4 mediated stimulation, which is also orchestrated by altering the RIP140 protein level (fig. 2b). We employed 2 animal models to support this conclusion, an MФRIP140KD transgenic mouse model where the RIP140 level in macrophages is reduced from birth, and a bone marrow transplant-generated macrophage replacement model where a nondegradable RIP140 mutant protein, Y3F, is introduced only during adult stages. Our data clearly show that the accumulated RIP140 protein level in adults is crucial to the dynamics of macrophage phenotype transition.

An unexpected finding in this in vivo wound experiment is that Arg1 gene expression is higher in Y3F-RIP140 mice than WT mice on day 10 (fig. 3d). It is important to note that gene regulation, especially in whole animals, can involve more complicated regulatory pathways. Y3FRIP140 could possibly exert additional effects that might have disturbed Arg1 gene regulation, causing a higher level of Arg1 gene expression in certain points of the woundhealing process, as was surprisingly observed on day 10 of this experiment. However, the expression of the 2 additional M2 markers, TGF $\beta$ and VEGF, and the M2 population analysis from FACS support the notion that prevent- 
ing RIP140 degradation (to an elevated RIP140 level) indeed delays wound healing, which involves inhibiting M1-M2 switch.

An interesting mechanistic finding is that the new M2suppressive function of RIP140 is achieved by its cytosolic form, exported from the nuclei in M2 cue-stimulated macrophages. RIP140 was initially identified as a coregulator of numerous transcription factors in the nucleus, but more studies have revealed that, in various cell types and stimulated by different signal inputs, nuclear RIP140 can be exported to the cytosol to regulate a wide variety of biological processes. Interestingly, all these events are highly cell type-selective and signal-specific. For instance, in mature adipocytes, the lipid signal stimulates its export to the cytosol to regulate AS160 to modulate insulin sensitivity [33]. In differentiated neurons, ER stress stimulates RIP140 export to the cytosol and then to ER, where it interacts with IP3R to modulate ER store calcium release, thereby protecting neurons from apoptosis [34]. In this study, we uncover RIP140 exported to the cytosol in M2 cue-stimulated macrophages to suppress the efficiency of M2 polarization. Clearly, in macrophages, RIP140 modulates the homeostasis of innate immunity via its dual functions in the M1 and M2 states. With this dual control in place, macrophages may not overreact to either stimulus. A fault in this control may result in devastating situations such as sepsis or delayed wound healing (when RIP140 cannot be degraded or exported in time). Surprisingly, the cytosolic RIP140 suppresses the M2 master regulator, pSTAT6, by targeting the regulatory step of a specific phosphatase of STAT6, PTP1B, in the ER/cytosol. This is achieved by its increasing association with cal- pain's regulatory subunit, CAPNS1, in IL-4-treated cells, to further activate calpain $1 / 2$ which cleaves the PTP1B precursor and renders its activation. Whether and how RIP140 association with CAPNS1 would alter calpain 1/2 activity must still to be determined. Activated PTP1B then efficiently dephosphorylates pSTAT6, thereby reducing M2 target gene activation.

PTP1B is a negative regulator for the insulin and leptin signaling pathways $[35,36]$. Currently, a PTP1B inhibitor, trodusquemine, and PTP1B-specific antisense oligonucleotides are in phase 2 clinical trials for treating diabetes or obesity $[37,38]$. Clearly, in innate immune cells, the expression level of RIP140 protein and its subcellular localization are critical to the maintenance of the functional plasticity of macrophages. The factors and molecules regulating its protein level and subcellular distribution can also be potential therapeutic targets. However, the mechanism underlying the M2 cue-stimulated (e.g. with IL-4 and IL-13) nuclear export of RIP140 has still to be determined. Furthermore, while RIP140 can be associated with the calpain regulatory subunit, CAPNS1, it remains unclear how this affects calpain activity. Rigorous biochemical studies are needed in order to understand the molecular details of activation of this specific protease complex.

\section{Acknowledgements}

This work was supported by DK54733, DK60521, DK5473311S, DK60521-12S1 and the Dean's Commitment and the Distinguished McKnight Professorship of University of Minnesota to L.-N.W. We thank Pei-Yao Liu and Raji Jasty for technical assistance.

\section{References}

1 Gordon S, Taylor PR: Monocyte and macrophage heterogeneity. Nat Rev Immunology 2005;5:953-964.

2 Galli SJ, Borregaard N, Wynn TA: Phenotypic and functional plasticity of cells of innate immunity: macrophages, mast cells and neutrophils. Nat Immunol 2011;12:1035-1044.

- 3 Sica A, Mantovani A: Macrophage plasticity and polarization: in vivo veritas. J Clin Invest 2012;122:787-795.

4 Mosser DM, Edwards JP: Exploring the full spectrum of macrophage activation. Nat Rev Immunol 2008;8:958-969.

$\checkmark 5$ Mantovani A, Biswas SK, Galdiero MR, Sica A, Locati M: Macrophage plasticity and polarization in tissue repair and remodelling. J Pathol 2013;229:176-185.
6 McNelis JC, Olefsky JM: Macrophages, immunity, and metabolic disease. Immunity 2014;41:36-48.

7 Stearns-Kurosawa DJ, Osuchowski MF, Valentine C, Kurosawa S, Remick DG: The pathogenesis of sepsis. Ann Rev Pathol 2011; 6:19-48.

8 Mantovani A: Cancer: inflaming metastasis. Nature 2009;457:36-37.

-9 Lawrence T, Natoli G: Transcriptional regulation of macrophage polarization: enabling diversity with identity. Nat Rev Immunol 2011; 11:750-761.
10 Liao X, Sharma N, Kapadia F, Zhou G, Lu Y, Hong H, Paruchuri K, Mahabeleshwar GH, Dalmas E, Venteclef N, Flask CA, Kim J, Doreian BW, Lu KQ, Kaestner KH, Hamik A, Clement K, Jain MK: Kruppel-like factor 4 regulates macrophage polarization. J Clin Invest 2011;121:2736-2749.

11 Krausgruber T, Blazek K, Smallie T, Alzabin S, Lockstone H, Sahgal N, Hussell T, Feldmann M, Udalova IA: Irf5 promotes inflammatory macrophage polarization and Th1-Th17 responses. Nat Immunol 2011;12:231-238.

12 Satoh T, Kidoya H, Naito H, Yamamoto M, Takemura N, Nakagawa K, Yoshioka Y, Morii E, Takakura N, Takeuchi O, Akira S: Critical role of Trib1 in differentiation of tissue-resident M2-like macrophages. Nature 2013;495: 524-528. 
13 Angus DC, van der Poll T: Severe sepsis and septic shock. N Engl J Med 2013;369:840-851.

14 Russell JA: Management of sepsis. N Engl J Med 2006;355:1699-1713.

15 Biswas SK, Lopez-Collazo E: Endotoxin tolerance: new mechanisms, molecules and clinical significance. Trends Immunol 2009;30: 475-487.

16 West MA, Heagy W: Endotoxin tolerance: a review. Crit Care Med 2002;30:S64-S73.

-17 Chan C, Li L, McCall CE, Yoza BK: Endotoxin tolerance disrupts chromatin remodeling and NF-kappaB transactivation at the IL-1beta promoter. J Immunol 2005; 175:461-468.

18 Nomura F, Akashi S, Sakao Y, Sato S, Kawai T, Matsumoto M, Nakanishi K, Kimoto M, Miyake K, Takeda K, Akira S: Cutting edge: endotoxin tolerance in mouse peritoneal macrophages correlates with down-regulation of surface Toll-like receptor 4 expression. J Immunol 2000;164:3476-3479.

-19 Kobayashi K, Hernandez LD, Galan JE, Janeway CA Jr, Medzhitov R, Flavell RA: IRAK-M is a negative regulator of Toll-like receptor signaling. Cell 2002;110:191-202.

20 Nautiyal J, Christian M, Parker MG: Distinct functions for RIP140 in development, inflammation, and metabolism. Trends Endocrinol Metab 2013;24:451-459.

21 Mostaqul Huq MD, Gupta P, Wei LN: Posttranslational modifications of nuclear co-repressor RIP140: a therapeutic target for metabolic diseases. Curr Med Chem 2008;15:386392.

-22 Ho PC, Tsui YC, Feng X, Greaves DR, Wei LN: NF-kappaB-mediated degradation of the coactivator RIP140 regulates inflammatory responses and contributes to endotoxin tolerance. Nat Immunol 2012;13:379-386.
23 Ho PC, Chang KC, Chuang YS, Wei LN: Cholesterol regulation of receptor-interacting protein 140 via microRNA-33 in inflammatory cytokine production. FASEB J 2011;25: 1758-1766.

24 Pena OM, Pistolic J, Raj D, Fjell CD, Hancock RE: Endotoxin tolerance represents a distinc tive state of alternative polarization (M2) in human mononuclear cells. J Immunol 2011; 186:7243-7254.

25 Porta C, Rimoldi M, Raes G, Brys L, Ghezzi P, Di Liberto D, Dieli F, Ghisletti S, Natoli G, De Baetselier P, Mantovani A, Sica A: Tolerance and M2 (alternative) macrophage polarization are related processes orchestrated by $\mathrm{p} 50$ nuclear factor kappaB. Proc Natl Acad Sci USA 2009; 106:14978-14983.

26 Nathan C, Ding A: Nonresolving inflammation. Cell 2010;140:871-882.

27 Chen J, Ivashkiv LB: IFN-gamma abrogates endotoxin tolerance by facilitating Toll-like receptor-induced chromatin remodeling. Proc Natl Acad Sci USA 2010;107:1943819443.

28 Lu X, Malumbres R, Shields B, Jiang X, Sarosiek KA, Natkunam Y, Tiganis T, Lossos IS PTP1B is a negative regulator of interleukin 4-induced STAT6 signaling. Blood 2008;112: 4098-4108.

29 Cortesio CL, Chan KT, Perrin BJ, Burton NO, Zhang S, Zhang ZY, Huttenlocher A: Calpain 2 and PTP1B function in a novel pathway with Src to regulate invadopodia dynamics and breast cancer cell invasion. J Cell Biol 2008;180:957-971.

30 Kuchay SM, Kim N, Grunz EA, Fay WP, Chishti AH: Double knockouts reveal that protein tyrosine phosphatase $1 \mathrm{~B}$ is a physiological target of calpain-1 in platelets. Mol Cell Biol 2007;27:6038-6052.
31 Arthur JS, Elce JS, Hegadorn C, Williams K, Greer PA: Disruption of the murine calpain small subunit gene, Capn4: calpain is essential for embryonic development but not for cell growth and division. Mol Cell Biol 2000;20: 4474-4481.

32 Zimmerman UJ, Boring L, Pak JH, Mukerjee $\mathrm{N}$, Wang KK: The calpain small subunit gene is essential: its inactivation results in embryonic lethality. IUBMB Life 2000;50:63-68.

33 Ho PC, Lin YW, Tsui YC, Gupta P, Wei LN: A negative regulatory pathway of GLUT4 trafficking in adipocyte: new function of RIP140 in the cytoplasm via AS160. Cell Metab 2009; 10:516-523.

34 Feng X, Krogh KA, Wu CY, Lin YW, Tsai HC, Thayer SA, Wei LN: Receptor-interacting protein 140 attenuates endoplasmic reticulum stress in neurons and protects against cell death. Nat Commun 2014;5:4487.

35 Chernoff J: Protein tyrosine phosphatases as negative regulators of mitogenic signaling. J Cell Physiol 1999;180:173-181.

36 Bence KK, Delibegovic M, Xue B, Gorgun CZ, Hotamisligil GS, Neel BG, Kahn BB: Neuronal PTP1B regulates body weight, adiposity and leptin action. Nat Med 2006;12:917-924.

>37 Lantz KA, Hart SG, Planey SL, Roitman MF Ruiz-White IA, Wolfe HR, McLane MP: Inhibition of PTP1B by trodusquemine (MSI1436) causes fat-specific weight loss in dietinduced obese mice. Obesity 2010;18:15161523

38 Cho H: Protein tyrosine phosphatase $1 \mathrm{~B}$ (PTP1B) and obesity. Vitam Horm 2013;91: 405-424. 